\title{
Electrically Conductive Adhesive Filled with Mixture of Silver Nano and Microparticles
}

\author{
Pavel Mach ${ }^{1}$, Radoslav Radev ${ }^{1}$, Alena Pietrikova ${ }^{2}$ \\ ${ }^{1}$ Czech Technical University in Prague, Faculty of Electrical Engineering \\ Technicka 2, 16627 Prague 6, Czech Republic \\ Email:mach@fel.cvut.cz, Phone: ++420 22435 2214, Fax: ++420 224363949 \\ ${ }^{2}$ Technical University of Kosice, Faculty of Electrical Engineering and Informatics \\ Letna 9, 04200 Kosice, Slovak Republic \\ Email: Alena.Pietrikova@tuke.sk, Phone: ++421 55 6023194, Fax: ++421 556023195
}

\begin{abstract}
Electrically conductive adhesive with isotropical electrical conductivity modified with addition of silver nanoparticles has been investigated. The electrical resistance, nonlinearity of a current vs. voltage characteristic and the tensile strength of adhesive joints formed of this adhesive have been measured. The specimens have also been aged at the temperature of $125^{\circ} \mathrm{C}$ and at the combined climate $80{ }^{\circ} \mathrm{C} / 80 \%$ relative humidity for 700 hours. It has been found that silver nanoparticles added into the electrically conductive adhesive cause decrease of its conductivity, increase of its nonlinearity and increase of the tensile strength.
\end{abstract}

\section{Introduction}

The resistance of adhesive joints formed of electrically conductive adhesives with isotropical electrical conductivity is higher in comparison with the resistance of soldered joints. The ratio of conductivity of adhesive vs. soldered joints is mostly higher than 1:5 or higher. The reason is that electrical conductivity of solder is dominantly influenced with a phonon-electron interaction, whereas electrical conductivity of adhesive is dominantly influenced with tunneling of electrons across barriers between conductive particles [1]. Electrical conductivity of soldered joints is also improved using different types of fluxes; electrically conductive adhesives are applied directly between a pad and a lead of a component without some additive substance improving contact quality.

There are different ways, which have been tested to improve electrical and mechanical properties of adhesive joints. The use of nanoparticles is not a new idea. Analysis of conductivity of electrically conductive adhesives filled with lead free solder nanoparticles is presented in [2]. Properties of electrically conductive adhesives filled with silver coated carbon nanotubes have been investigated in [3]. Effect of silver nanowires on electrical conductance of system composed of silver particles has been described in [4]. The resistivity of polymeric conductive adhesives filled with nano-sized silver particles has been investigated in [5].

Basic parameters used for description of quality of adhesive joints are the joint resistance and the tensile strength, usually. We have added these measurements with the measurement of nonlinearity of a current vs. voltage characteristic.
With respect to the structure of electrically conductive adhesives with isotropical electrical conductivity the dominant conductive mechanism is tunneling between neighboring filler particles and tunneling between these particles and a pad on PCB or a lead of a component. Tunneling mechanism is nonlinear and therefore the measurement of the adhesive joint current vs. voltage characteristic nonlinearity can be useful tool for evaluation of joint quality. Influence of addition of silver nanoparticles into the standard type adhesive on the joint resistance has been already investigated. Knowledge of such information can be interesting with respect to the possible application of adhesives in a high frequency range, where joint nonlinearity can cause problems following of generation of intermodulatoin signals on such nonlinearity.

\section{Theoretical Background}

The total electrical resistance of an adhesive joint consists of following components:

- Electrical resistance of filler particles.

- Electrical resistance of contacts between filler particles.

- Electrical resistance of a contact between filler particles and a pad on a PCB.

- Electrical resistance of a contact between filler particles and a lead of a component.

The value of the electrical resistance of filler particles is substantially lower in comparison with the value of the contact resistance between filler particles and between filler particles and a pad or a lead of a component if $\mathrm{Ag}$ filler particles are used. If the filler particles are plastic balls covered with a thin conductive metal film, the filler particle resistance can be comparable with the contact resistance. However, such the particles are used for adhesives with anisotropical electrical conductivity only and we have tested adhesive with isotropical conductivity filled with Ag flakes.

The contact resistance between two particles of the filler consists of following parts:

- The constriction resistance.

- The tunnel resistance.

The total contact resistance can be calculated as a sum of these two components. The reason of the constriction resistance is as follows: let us assume that two semiinfinity conductive segments are mutually connected by only a small circle contact with the diameter of $2 a$. In the area of a contact the current lines taper and the result 
of this effect is an increase of the resistance. If the conductive segments have the same area and if they are fabricated of the same material or of materials with the same resistivity $\rho$, the constriction resistance $R_{C}$ can be written as:

$$
R_{C}=\frac{\rho}{2 a}
$$

This formula has been derived for a small circle contact with the diameter $2 a$ joining two infinity bodies; however, it is also possible to use it for calculation of the constriction resistance between two flakes with sufficient accuracy [1].

The second component of the contact resistance is the tunnel resistance. This resistance depends strongly on the thickness and quality of an insulating barrier. The tunnel resistance $R_{T}$ is calculated using the tunnel resistivity $\sigma\left(\Omega \mathrm{m}^{2}\right)$. The resistance of a tunnel contact with the diameter of $2 a$ can be calculated as follows:

$$
R_{T}=\frac{\sigma}{\pi \cdot a^{2}}
$$

The tunnel resistivity can be calculated using a formula:

$$
\begin{aligned}
& \sigma=\sigma\left(s, \Phi, \varepsilon_{r}\right)=\frac{10^{-22}}{2} \frac{A^{2}}{1+A B} e^{A B} \\
& A=7,32 \cdot 10^{5}\left(s-\frac{7,2}{\Phi}\right) \\
& B=1,265 \cdot 10^{-6} \sqrt{\Phi-\frac{10}{s \varepsilon_{r}}}
\end{aligned}
$$

Where: $\quad s \ldots$ thickness of a tunnel barrier, $\Phi$... output work of an electron from a metal, $\varepsilon_{r} \ldots$ dielectric constant of the tunnel barrier material.

The total contact resistance between filler particles is a sum of the constriction and the tunnel resistance.

$$
R=R_{C}+R_{T}=\frac{\rho}{2 a}+\frac{\sigma}{\pi \cdot a^{2}}
$$

The next part of the contact resistance is the resistance between the filler particles and the pad or lead of a component. Quality of these contacts depends on the materials of the filler particles and the pad or lead. If the pad or lead are fabricated, or if their surface finish is, of such metals as $\mathrm{Cu}, \mathrm{Sn}, \mathrm{Ni}$ or $\mathrm{Pb}$ or of other metals with low electrochemical potential, the contact between these metals and metals with the high electrochemical potential such as Ag, Au or Pt can create a galvanic a voltaic cell if water is present. This cell can cause electrochemical corrosion, which decreases quality of the contact. Therefore it is recommended to use for surface finish of pads or leads of components a metal with similar electrochemical potential like Ag.

For contact between flakes it is hard to calculate a contact area. However, it is assumed that the contact areas are larger than those between balls. This assumption can be confirmed using SEM analyzis. Therefore the constriction resistance between flakes can be neglected and the total contact resistance can be described with the tunnel resistance only.

$$
R=R_{T}=\frac{\sigma}{A}
$$

Where: $A$... contact area between flakes.

The equations (1) to (7) describe properties of contacts between conductive balls and between conductive flakes used as filler of adhesive. If the total adhesive joint resistance should be calculated, the conductive net formed of filler particles has to be simulated, and the contacts in contact points are described using equations (6) or (7). The resistance of filler particles is small in comparison with the contact resistance and therefore it can be neglected for calculation of the total resistance.

If conductive nanoparticles (grains or balls) are added into electrically conductive adhesive with isotropical electrical conductivity, they can be distributed among filler particles of adhesive as follows:

- The nanoparticles are not aggregated and are randomly located between flakes.

- The nanoparticles are partially aggregated and create additional conductive bridges between flakes.

The total electrical resistance of the tunnel joint formed of electrically conductive adhesive with isotropical conductivity, where flakes are used as the filler, is dominantly influenced by the tunnel resistance between flakes. Obviously, the tunnel resistance between flakes and pad and between flakes and component lead are also significant. When nanoparticles are added into the adhesive and are not aggregated, they are randomly distributed between flakes. The total number of contacts in conductive net increases and the total resistance of the conductive net too.

On the other hand, if the nanoparticles, after addition into electrically conductive adhesive, are able to aggregate and to create additional conductive bridges, parallel with contacts between flakes, the joint resistance decreases.

Different theories have been developed for description of nonlinearity of a current vs. voltage characteristic. Theory of nonlinearity, based on an assumption that nonlinearity of current vs. voltage characteristic is dominantly influenced by thermal movement of vacancies inside the material has been presented Zhigalsky [6].

Other theory of nonlinearity is based on an assumption that nonlinearity of current vs. voltage characteristic is dominantly caused by potential barriers inside the material. According to this theory the current flowing through the potential barrier can be calculated using the equation:

$$
I_{T}=2 N_{T} A \exp \left(-\frac{e \Phi_{I}}{k T}\right) \cdot\left[\frac{e U}{k T}+\frac{1}{3 !}\left(\frac{e U}{k T}\right)^{3}+\frac{1}{5 !}\left(\frac{e U}{k T}\right)^{5}+\ldots\right]
$$


Where $\mathrm{N}_{\mathrm{T}} \ldots$ total number of barriers, A ... constant dependent on the material, e ... charge of an electron, $\Phi_{i} \ldots$ high of the potential barrier, $k$... Boltzmann constant, $\mathrm{T}$... temperature, $\mathrm{U}$... voltage across the joint.

\section{Experimental}

Adhesive bonds have been formed by adhesive assembly of resistors with zero resistance (jumpers, 1206 0R0 for adhesive assembly) on test boards (FR4, Cu $40 \mu \mathrm{m})$.

One-component isotropically electrically conductive adhesive composed of bisphenol-epoxy resin and silver flakes (dimensions 6 to $8 \mu \mathrm{m}$ ) has been used as the basic adhesive for experiments. Concentration of silver flakes in this adhesive has been $75 \%$ (wt.). This adhesive has been modified by addition of silver nanoparticles.

Nanoparticles have been grains of 3 dimensions: 3 to $55 \mathrm{~nm}, 6$ to $8 \mathrm{~nm}$ and 80 to $100 \mathrm{~nm}$.

Addition of nanoparticles into the basic adhesive has been carried out by following ways:

- A part of silver flakes has been substituted with nanoparticles to preserve the constant contents of silver in adhesive.

- The total amount of silver in adhesive has been increased by addition of nanoparticles,

Following modifications of the basic adhesive have been used for experiments:

Tab. 2 Modifications of the basic adhesive under test. Basic adhesive: ICA, bisphenoí-epoxy resin, silver flakes 6 to $8 \mu \mathrm{m}, 75 \%$ wt.

\begin{tabular}{|c|c|c|c|}
\hline $\begin{array}{c}\text { Sample } \\
\#\end{array}$ & $\begin{array}{c}\text { Nanoparticles } \\
\text { dimensions } \\
(\mathrm{nm})\end{array}$ & $\begin{array}{l}\text { Concent- } \\
\text { ration } \\
\% \text { (wt.) }\end{array}$ & Processing \\
\hline s1 & $(3-55)$ & 10 & \multirow{3}{*}{ 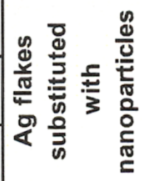 } \\
\hline S2 & $(3-55)$ & 20 & \\
\hline S3 & $(3-55)$ & 30 & \\
\hline S4 & $(80-100)$ & 3,8 & \multirow{4}{*}{ 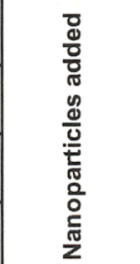 } \\
\hline S5 & $(6-8)$ & 3,8 & \\
\hline S6 & $(80-100)$ & 7,4 & \\
\hline S7 & $(6-8)$ & 7,4 & \\
\hline
\end{tabular}

Sets of 42 adhesive joints have been formed of every sample of modified adhesive and the same number of adhesive joints has been formed of the basic adhesive to have a standard for investigation of properties changes caused with addition of nanoparticles.

\section{Measurement of electrical and mechanical parameters}

The electrical resistance has been measured using a MCP TH 2818 Automatic Component Analyzer.
Nonlinearity of the current vs. voltage characteristic of adhesive joints has been measured using equipment designed and realized at the Department of Electrotechnology. The principle of the measurement is the evaluation of a level of intermodulation products generated by nonlinearity of the adhesive joint, which is powered with two sinusoidal signals with different frequencies.

The tensile strength has been measured using a pulloff test. KERN MH10K10 digital tester has been used for this measurement. The arrangement for the measurement of the tensile strength is shown in Fig. 1.

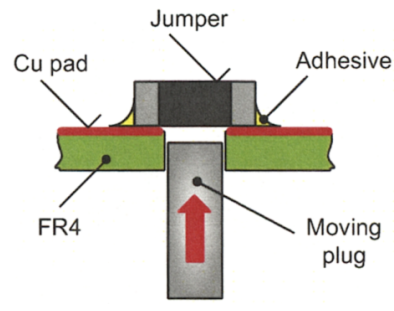

Fig. 1 Measurement of the tensile strength of adhesive joints

Following climatic load has been applied on adhesive joints formed of the samples of modified adhesive marked S1 to S3:

- Temperature $125^{\circ} \mathrm{C}$ for 700 hours.

- Combined climatic load $80{ }^{\circ} \mathrm{C} / 80 \%$ RH for 700 hours.

\section{Results and discussion}

The starting values of the resistance, nonlinearity of the current vs. voltage characteristic and tensile strength of adhesive joints are shown in Fig. 2, Fig. 3 and Fig. 4.

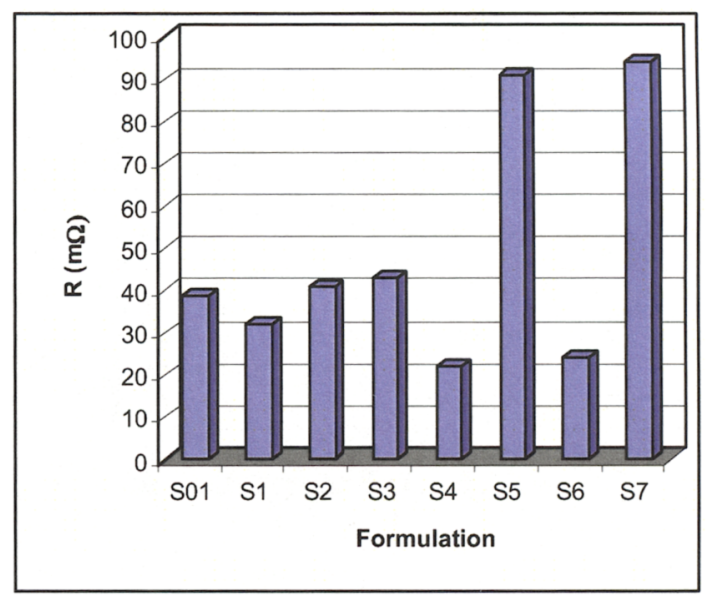

Fig. 2 Resistance of adhesive joints formed of modified adhesives, $\mathrm{S} 01$ is the basic adhesive used for modification

Changes of the resistance, nonlinearity and tensile strength of the adhesive joints after thermal ageing at the temperature of $125^{\circ} \mathrm{C}$ for 700 hours are shown in Fig. 5, Fig. 6 and Fig. 7. 


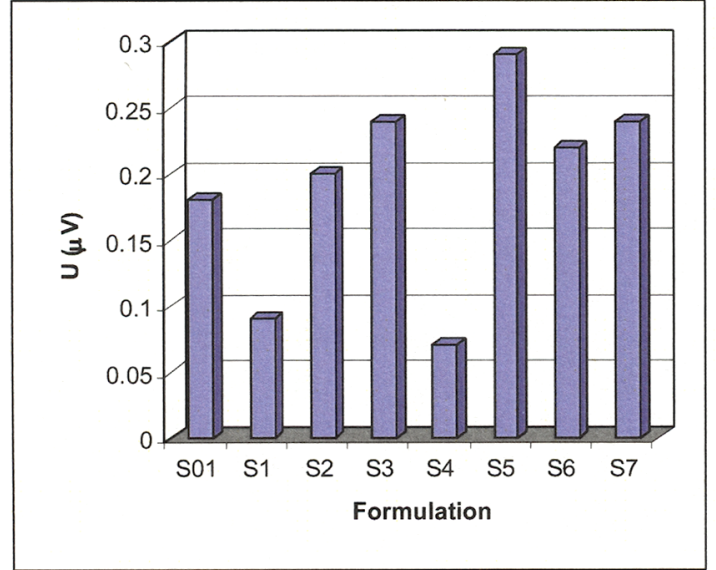

Fig. 3 Nonlinearity of adhesive joints formed of modified adhesives, $\mathrm{S} 01$ is the basic adhesive used for modification

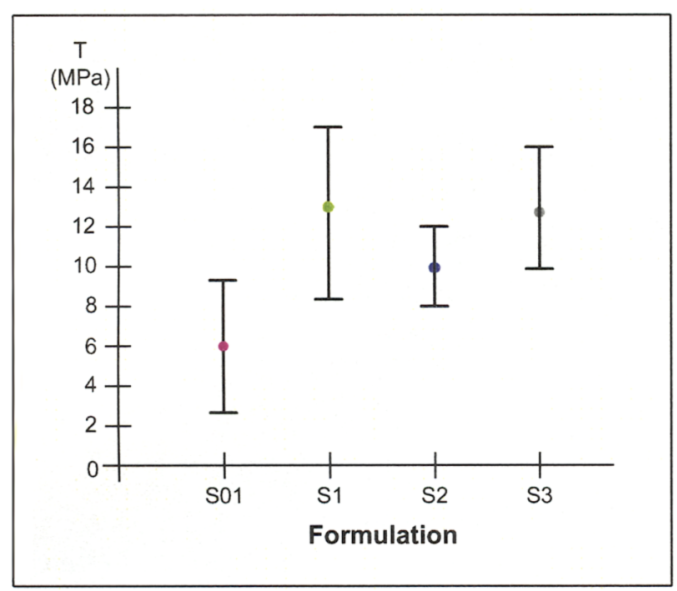

Fig. 4 Tensile strength of modified adhesives. S01 is the basic adhesive used for modification

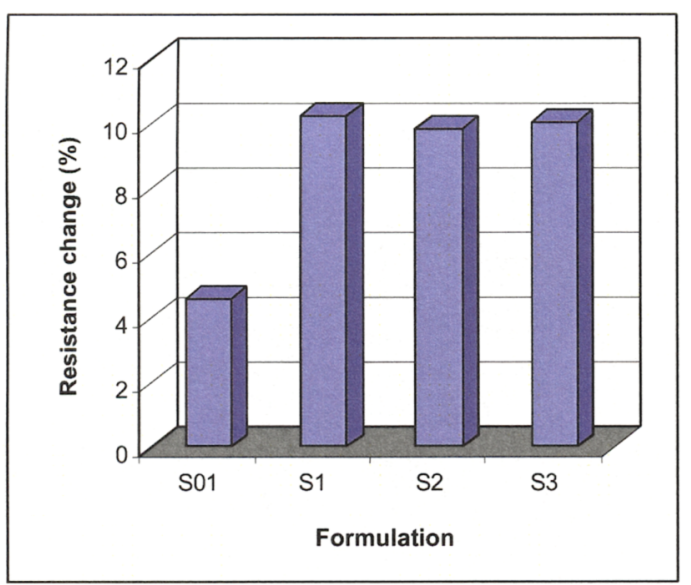

Fig. 5 Resistance change of adhesive joints formed of modified adhesives after thermal ageing $\left(125^{\circ} \mathrm{C}, 700\right.$ hours $)$. $\mathrm{S} 01$ is the basic adhesive used for modification

Changes of the resistance, nonlinearity and tensile strength of the adhesive joints after combined thermal/humidity ageing at the temperature of $80{ }^{\circ} \mathrm{C}$ and the relative humidity of $80 \%$ for 700 hours are shown in Fig. 8, Fig. 9 and Fig. 10.

The increase of the joints resistance after addition of silver nanoparticles into the adhesive (Fig. 2) is caused by additional contacts, which are created by nanoparticles in conductive net of adhesive. When the same weight of nanoparticles is added, the frequency of these contacts is the higher, the lower are dimensions of nanoparticles.

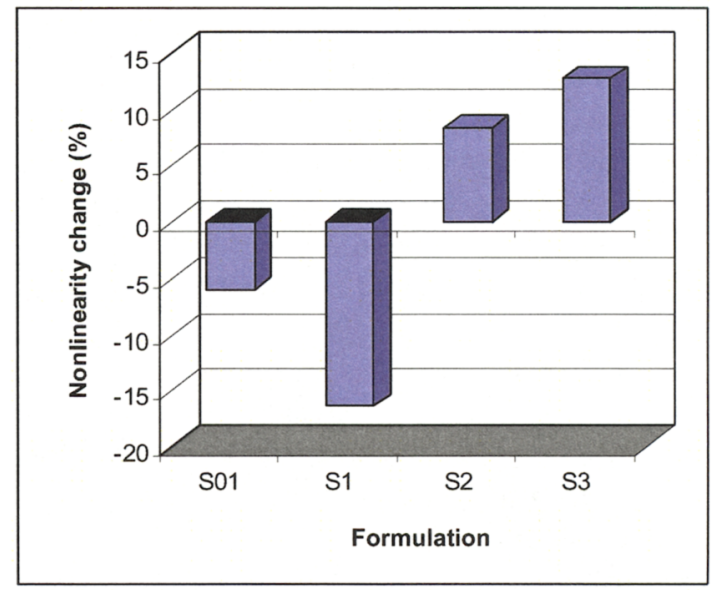

Fig. 6 Nonlinearity change of adhesive joints formed of modified adhesives after thermal ageing $\left(125^{\circ} \mathrm{C}, 700\right.$ hours $)$. $\mathrm{S} 01$ is the basic adhesive used for modification

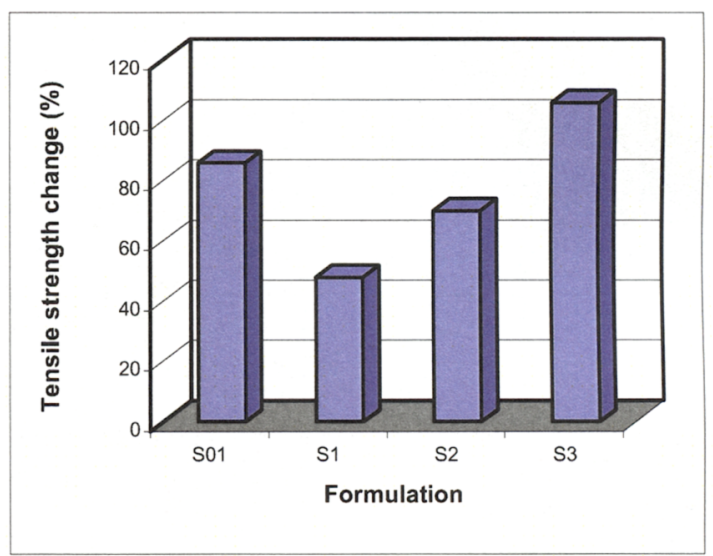

Fig. 7 Tensile strength change of modified adhesives after thermal ageing $\left(125^{\circ} \mathrm{C}, 700\right.$ hours $), \mathrm{S} 01$ is the basic adhesive used for modification

Therefore the increase of the resistance is higher for nanoparticles of lower dimensions (see formulations S5 and S7 in Fig. 2).

These "new" contacts have, as for the resistance, a constriction part and a tunnelling part, because they are created by particles of small dimensions. Both these parts are nonlinear. Therefore addition of nanoparticles causes mostly increase of the joint nonlinearity (see Fig. 3).

Exceptions (modifications S1 and S4) are samples with a small amount of nanoparticles added and the nonlinearity decrease can be joined with the decrease of the resistivity in comparison with the basic adhesive (see 
Fig. 2). The significant information is that the decrease of the resistivity has been found for "big" nanoparticles and low concentrations only (see Tab. 1).

It has been found that the tensile strength of adhesives modified with the nanoparticles is higher in comparison with the basic adhesive (see Fig. 4).

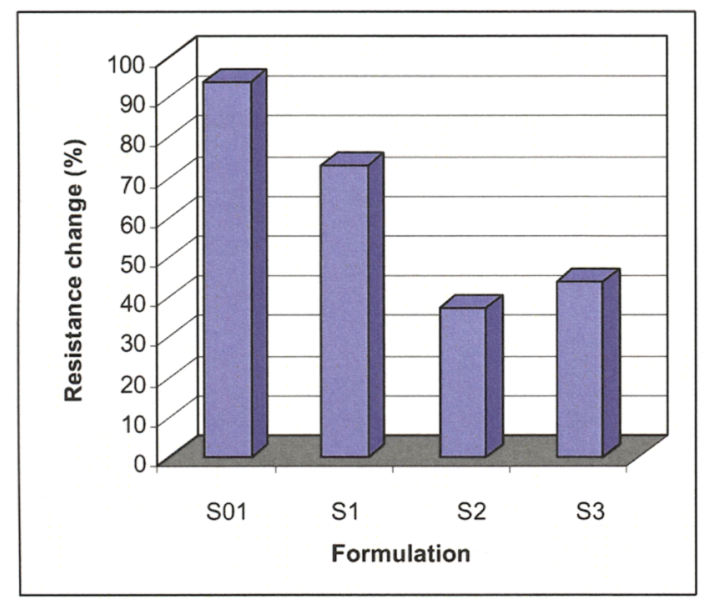

Fig. 8 Resistance change of adhesive joints formed of modified adhesives after combined thermal/humidity ageing $\left(80^{\circ} \mathrm{C}, 80 \% \mathrm{RH}, 700\right.$ hours). S01 is the basic adhesive used for modification

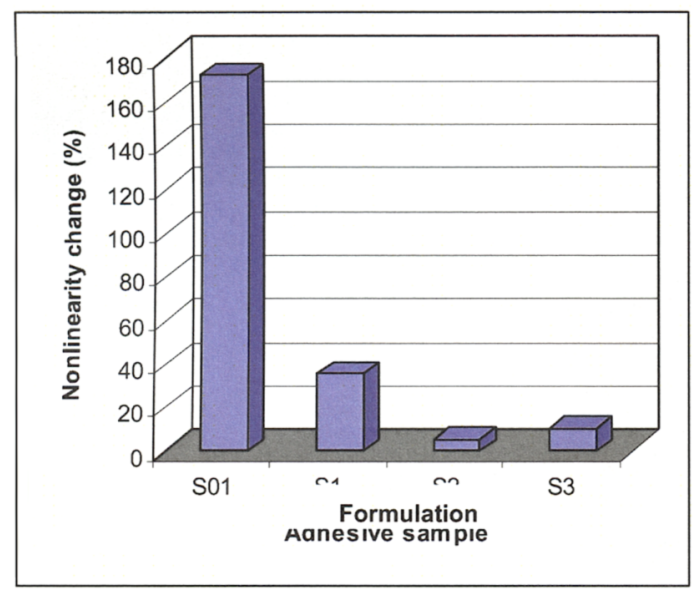

Fig. 9 Nonlinearity change of adhesive joints formed of modified adhesives after combined thermal/humidity ageing ( $80{ }^{\circ} \mathrm{C}, 80 \% \mathrm{RH}, 700$ hours). $\mathrm{S} 01$ is the basic adhesive used for modification

Such the increase could be assumed when the adhesives would be filled with carbon nanotubes or silver nanowires. However, it has been found that also nanoparticles can improve mechanical properties of adhesives. The reason is that the chemical bonds between nanoparticles and the resin are stronger than bonds in the resin.

The resistance of adhesive joints has increased after thermal aging due to partial damage of contacts between the conductive particles.

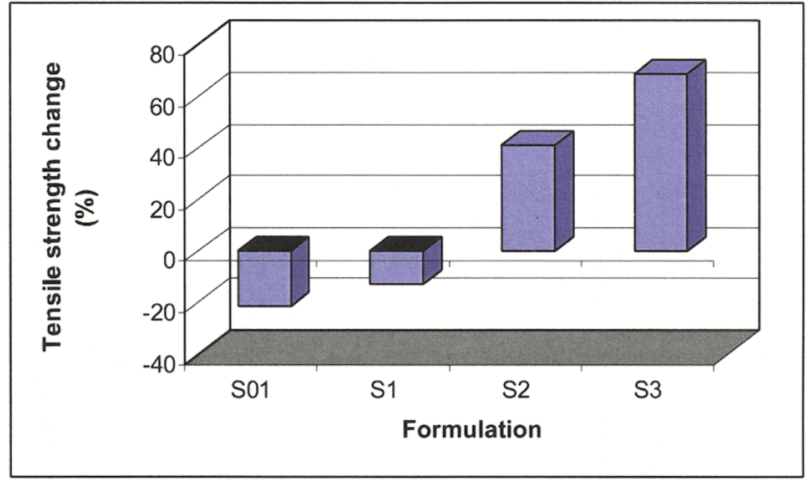

Fig. 10 Tensile strength change of modified adhesives after combined thermal/humidity ageing $\left(80{ }^{\circ} \mathrm{C}, 80 \% \mathrm{RH}, 700\right.$ hours). $\mathrm{S} 01$ is the basic adhesive used for modification

This damage has been caused by oxidation or other chemical process. It has been found (see Fig. 5) that the higher is the contents of nanoparticles, the higher is increase of nonlinearity after thermal ageing. The reason is the increase of the total number of contacts in the conductive net after adhesive modification.

Nonlinearity change of the adhesive joints after thermal ageing is negative for the basic adhesive and negative and positive for different formulations with the nanoparticles (Fig. 6). Such the result can be explained by different conductivity mechanisms, which dominate in different formulations after the thermal ageing.

Increase of the tensile strength for all measured formulations (Fig. 7) is caused with additional hardening of the adhesive during thermal ageing.

Positive influence of nanoparticles, added into the electrically conductive adhesive, on the resistance and nonlinearity of adhesive, has been found for combined climatic ageing. It has been detected lower increase of the resistance (Fig. 8) and significantly lower increase of nonlinearity (Fig. 9) after combined thermal/humidity ageing for the samples with nanoparticles in comparison with the samples without them. The reason is that the contacts between nanoparticles have lower dimensions in comparison with the contacts between flakes. Therefore the contact pressure is higher for these contacts and they are more resistive against damage with chemical layer created as a consequence of combined climatic ageing.

Tensile strength of the formulation S1 has decreased after combined climatic ageing (but this decrease has been lower in comparison with the decrease of the tensile strength of the basic adhesive), whereas the tensile strength of formulations S2 and S3 has increased (see Fig. 10). The reason is as follows: the adhesive have been additionally hardened during this type of ageing and the bonds between the nanoparticles and adhesive are stronger in comparison with the bonds between flakes and the adhesive. Therefore the penetration of water molecules into the adhesive is more limited in formulations with nanoparticles than in the standard adhesive. 


\section{Conclusions}

The resistance, nonlinearity and tensile strength of the adhesive joints formed of ICA modified with silver nanoparticles of different dimensions have been examined. It has been found that the nanoparticles do not improve the electrical resistance and nonlinearity of the joints. The reason is that the total number of contacts between the conductive particles increases after addition of the nanoparticles into the basic adhesive. It has also been found that addition of nanoparticles improves the tensile strength and the resistance against combined climatic ageing of the adhesive. The reason is that the contact areas between nanoparticles are substantially smaller in comparison with the contact areas between flakes. Therefore the contact pressure between nanoparticles is higher than between flakes and the contacts are more resistant to the external load.

\section{Acknowledgments}

The work has been carried out as a part of a project "Diagnostics of Materials", number MSM6840770021

\section{References}

1. Su, B.: Electrical, Thermomechanical and Reliability Modeling of Electrically Conductive Adhesives. Dissertation. Georgia Institute of Technology, May 2006, pp. 64-65.

2. Verma, S.CH., Guan, W., Liu, J.: "Flip-Chip Interconnection Using Anisotropic Conductive with Lead Free Nano-Solder Particles", Proc. $1^{\text {st }}$ Electronics Systemintegration Technology Conference, 2006, vol.1, pp. 282-286.

3. Wu, H.P., Wu, X.J., Ge, M.Y., Zhang, G.Q., Wang, Y.W., Jiang, J.: "Properties investigation on isotropical conductive adhesives filled with silver coated carbon nanotubes." Composites Science and Technology: Vol. 67, Issue 6 (2007), pp. 1182-1186.

4. Chang, Ch., Li, W., Ruilin, L., Guohua, J., Haojie, Y., Tao, Ch.: "Effect of silver nanowires on electrical conductance of system composed of silver particles." Journal of Materials Science. Vol. 42, No 9 (2007). pp. 3172-3176

5. Lee, H. H., Chou, K. S., Shih Z. W.: "Effect of nanosized silver particles on the resistivity of polymeric conductive adhesives." International Journal of Adhesion and Adhesives, Vol. 25, Issue 5 (2005), pp. 437-441

6. Zhigalski, G.P.: "Noise $1 / \mathrm{f}$ and nonlinear effects in thin metal films (in Russian)." Uspěchy fyzičeskich nauk. Vol. 6 (1997), pp. 623-647. 